\title{
Changes in diagnoses before admission to a specialist medium secure unit
}

\author{
Amrith Shetty, Dilum Jayawickrama, ${ }^{2}$ Pamela J. Taylor ${ }^{3}$
}

The Psychiatrist (2012), 36, 404-408, doi: 10.1192/pb.bp.111.036004

${ }^{1}$ Betsi Cadwaladr University Health Board, Bangor; ${ }^{2}$ Partnerships in Care, Llanarth Court, ${ }^{3}$ School of Medicine, Cardiff University

Correspondence to Amrith Shetty (amrith.shetty@wales.nhs.uk)

First received 9 Jul 2011, final revision 22 Apr 2012, accepted 14 May 2012

\begin{abstract}
Aims and method There is evidence that changing diagnoses may be an important factor preceding homicide, but there is little literature on diagnostic antecedents to admission to specialist secure units after violent behaviour. Our aim was to establish the frequency of a history of changing diagnoses in patients in a UK specialist unit, and to explore the characteristics of these patients.
\end{abstract}

Results In total, 38 of 42 study participants had prior contact with psychiatric services. Just over $40 \%$ (16 of the 38 ) had had their diagnosis changed three or more times. All those who had major changes in their diagnosis had received a diagnosis of a psychotic illness at some point prior to the secure unit admission, but then had it withdrawn, only to be restored after prolonged assessment in the secure unit. Personality disorder and substance misuse comorbidity was common in this group; however, non-psychotic diagnoses were seen as more important than psychotic diagnoses by general services.

Clinical implications Changes in diagnosis between first presentation to psychiatric services and admission to a medium-security unit were more common than would be expected from reports in the general literature. They are a testimony to the difficulties experienced by service providers in delivering a consistent service. This needs to be studied further.

\section{Declaration of interest None.}

Some degree of diagnostic variability is seen routinely in clinical practice and can be attributed to different factors including progression of the illness or diagnostic error. It has been observed, however, that, in England, in several cases in which a homicide had been committed by a person who was previously in contact with psychiatric services, an independent homicide inquiry (as required by the Department of Health ${ }^{1}$ ) showed that there had been substantial diagnostic changes prior to the homicide. ${ }^{2}$ In the cases, for example, of Sharon Campbell and Christopher Clunis, early diagnoses of schizophrenia had been changed to a diagnosis of personality disorder, reverting after the killing to a diagnosis of schizophrenia. ${ }^{3,4}$ This may have had a profound effect on treatment, which is illustrated by a much later Welsh case: ${ }^{5}$

Ms A's contact with mental health services . . . can be divided into two periods: 1992-1998 when she was given a diagnosis of schizophrenia and treated with anti-psychotics and followed up by services. And in 2003-2005 when she was given a diagnosis of borderline personality disorder and not given continued treatment or followed up. (p. 2)

A literature review undertaken first using terms relating to diagnosis and forensic mental health services, and then adding terms relating to diagnostic stability/change revealed limited evidence in published literature about whether a history of changing diagnosis is a feature of people referred to forensic mental health services. Melzer et $a l$, in a study looking at access to medium psychiatric care in England and Wales, point out that there was good level of agreement between the referrer and the assessor in terms of primary diagnosis but do not elaborate on this. ${ }^{6}$ Other studies into patients in forensic units have not specifically tackled this issue. Our aim, therefore, was to explore the questions of how commonly major diagnostic change had preceded a behavioural event - usually violence to others of a nature sufficiently serious to warrant admission to a medium secure unit, and what factors were associated with such diagnostic change. A tentative hypothesis was that changing diagnoses would be associated with challenging behaviour, defined by repeated aggression or antisocial behaviour needing prolonged levels of care over and above routine care.

\section{Method}

Permission was sought to study a complete cohort of patients resident at any time over a 2-year period in the South Wales forensic medium secure unit - Caswell Clinic. This unit provides a forensic mental health service to an estimated population of 2.2 million, and a national service for long-term medium-security patients for the whole of 
Wales. It offers 64 beds in 5 wards and caters for both male and female patients. Facilities available are a psychiatric intensive care unit, only-male and only-female ward environments and a rehabilitation ward. The average length of stay is about 2 years. Referrals are received from the prison service, criminal justice system, general psychiatric hospitals and community-based agencies such as Social Services. The clinic also undertakes the work of rehabilitating people who have received treatment in high-security hospitals. Permission was granted by unit staff on the basis that the work proposed fitted criteria for a service evaluation. ${ }^{7}$ The local research ethics committee also expressed satisfaction that this was the case.

All patients who were 18 and older who had been resident in the Caswell Clinic under a first admission between 1 February 2006 and 31 January 2008 were included. A checklist was designed to facilitate recording of both categorical and narrative data.

For the purpose of the evaluation, multiple diagnostic changes were defined as three or more changes in diagnoses. 'Challenging behaviour' was defined as repeated aggressive or violent behaviour posing a particular challenge to the service from which the patient was referred because the behaviour was very frightening or disturbing to other patients, or perceived as likely to overwhelm the service because of the disproportionate amount of time needed to secure the patient, or because of the related fiscal costs of doing so. Reasons for referral were documented as narrative. All other data were simple factual points including demographics and nature of index offence, if any.

Information was collected entirely from clinical records. This included a review of all the data held about the patient at Caswell Clinic including case-note entries, referral details, previous psychiatric assessments or treatments, court reports, tribunal reports and criminal history details. A pilot study was carried out using the records of three patients in the clinic who were not to be included in the study, to check for feasibility. Data were entered onto the final checklist by two clinically trained reviewers, with eight of the cases being reviewed independently by both reviewers to check interrater reliability. There was full agreement on the ratings of all items in these eight cases. The data were entered onto an electronic database, designed using Microsoft Access 2007 on Windows and analysed using Statistical Product and Service Solutions (SPSS, version 11 for Windows).

\section{Results}

\section{General characteristics}

After excluding repeat admissions, a total of 42 patients had been resident in Caswell Clinic at some time between 1 February 2006 and 31 January 2008. Of these, 35 (83\%) were men and 7 (17\%) were women. The mean age of the male patients was 38 years $($ s.d. $=11.5$, range 44 ) and of the female patients 42 years (s.d. $=11.8$, range 31 ). The average length of stay in the clinic during the time studied was 1.8 years (s.d. $=1.8$, range 7 ).

Of the 42 patients, 9 (21.4\%) were admitted under civil sections of the Mental Health Act 1983 (Part 2), 32 (76.2\%) were admitted under the criminal part of the Mental Health Act (Part 3) and 1 was a voluntary patient. In total, 16 of the patients were referred from prison, 7 from general psychiatry services, 16 from other forensic services including medium secure and high secure services, and 3 were from a police custody suite.

\section{Reasons for referral and nature of offending or behavioural disorder}

Figure 1 shows the reasons for referral to the service. The largest group, about a third of the sample, consisted of people who had presented with challenging behaviour (categories 1-4 in the figure) while an in-patient elsewhere. One further group made up about a fifth of the sample, namely patients being assessed prior to appearing in court. Categories 6-8 contained the fewest number of patients.

\section{Diagnostic variables}

Following assessment at Caswell Clinic, 33 (78.6\%) of the patients had been given a primary diagnosis of paranoid schizophrenia, 2 schizoaffective disorder, 1 delusional disorder, 4 major depressive disorder (1 with psychotic symptoms), 1 bipolar affective disorder, 1 post-traumatic stress disorder and 1 obsessive-compulsive disorder. In terms of secondary diagnoses, drug misuse (harmful use/ dependence) (6 patients) and personality disorder (7 patients) were by far the most common. Patients in the

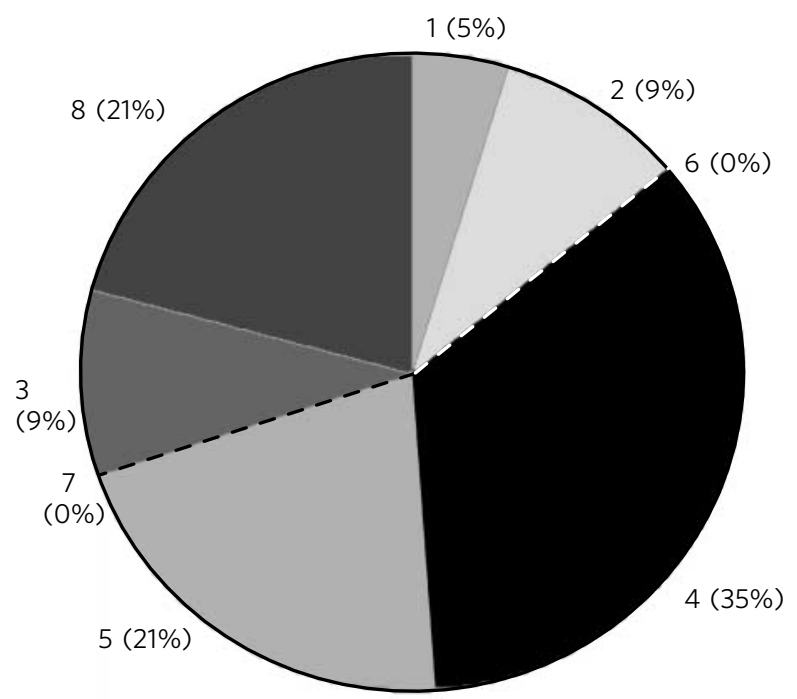

1. Repeated harm only to self while resident in an institution.

2. Repeated harm only to others while resident in an institution.

3. Both harm to self and others while resident in an institution.

4. Other challenging behaviour while resident in an institution.

5. Assessment for the court/defence after a charge/conviction for an index offence with a view to admission to Caswell.

6. As above with admission always viewed as unlikely at the time of referral - no individuals in this category in our study.

7. For a place on the route out of higher security - no individuals in this category in our study.

8. Other (specify).

Fig 1 Main reasons for referral. 
paranoid schizophrenia group were less likely than patients with other primary illness diagnoses to have at least one additional diagnosis (paranoid schizophrenia 8/33: (24\%) compared with $4 / 5$ (80\%) patients with other diagnoses.

In total, 16 of the 38 patients with previous contact with psychiatric services had had their diagnosis changed over time. Three examples illustrate some of the patterns of change of diagnoses.

In patient one (Box 1), anxiety had been the main initial presenting feature, perhaps with the patient himself still able to conceal the frightening experiences that made him so anxious. He then entered a pattern not unlike that seen in homicide inquiry cases, where his psychosis was

\section{Box 1 Patient one}

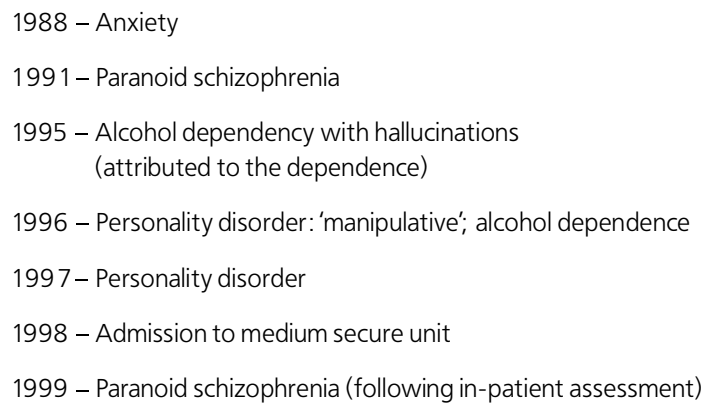

\section{Box 2 Patient two}

1999 - Drug dependency, attention-deficit hyperactivity disorder

2000 - Personality disorder, perhaps psychosis

2004 - Bipolar II disorder

2004 - Intermittent paranoid psychosis, significant substance misuse

2005 - Admission to medium secure unit

2005 - Paranoid schizophrenia

\section{Box 3 Patient three}

1985 - Paranoid schizophrenia

1992 - Treatment-resistant schizophrenia

1998 - 'All his problems related to glue sniffing': discharged from services

2000 - 'Not fit for treatment ... not within remit of Mental Health Act'

2001 - Hypomania

2001 - Thought disordered

2005 - Medium secure unit admission

2005 - Paranoid schizophrenia and mental and behavioural disorders due to volatile solvent use apparently rejected or explained away by clinicians, only to be confirmed after his offence and admission to the secure unit.

For patient two (Box 2) there had been no recognition of the possibility of psychosis for over 5 years after presentation. Once this was recognised, changes in diagnosis were within the psychotic spectrum.

Patient three (Box 3) had received a clear initial diagnosis of schizophrenia, but over time this was withdrawn and substituted with a diagnosis of substance misuse. Although the diagnosis of personality disorder was never invoked, he had some similarities to patients one and two and was explicitly rejected from services because he was seen as failing to meet criteria for inclusion into services. In the secure unit both diagnoses were accepted.

The one thing all three individuals in these examples had in common was that a diagnosis of some form of psychosis had been given at some point during the course of their illness prior to the medium secure unit admission, and rejected at some point before its restoration after an offence and prolonged assessment during detention in mediumsecurity hospital conditions.

Examination for any association between diagnostic change and having presented with challenging behaviour over time is shown in Table 1. There was no significant difference between changing and stable diagnostic groups in the occurrence of challenging behaviour, but there was a hint that challenging behaviour was less likely in the changing diagnostic group, contrary to our prediction. Table 2 shows that patients were slightly more likely than not to have had a mental healthcare plan in place prior to their admission, regardless of whether they were in the changing diagnosis group or not.

All six of the patients with a dual diagnosis of substance misuse and mental disorder had three or more changes in diagnosis (100\%) compared with only $31 \%$ (10 out of 32 ) of the patients without a substance misuse diagnosis.

Table 1 Multiple changes in diagnosis and challenging behaviour $(n=38)$

Challenging behaviour, $n(\%)$

\begin{tabular}{lll} 
& \multicolumn{2}{c}{ Challenging behaviour, $n(\%)$} \\
\cline { 2 - 3 } & \multicolumn{1}{c}{ Yes } & No \\
\hline $\begin{array}{lll}\text { Changing diagnosis } \\
\text { Yes }\end{array}$ & $6(33.3)^{\mathrm{a}}$ & $10(50)^{\mathrm{a}}$ \\
No & $12(66.6)$ & $10(50)$ \\
\hline
\end{tabular}

a. Not statistically significant: $\chi^{2}=0.504, P=0.477$.

Table 2 Multiple changes in diagnosis and whether a care plan was in place before admission to unit $(n=38)$

\begin{tabular}{llr} 
& \multicolumn{2}{c}{ Care plan, $n(\%)$} \\
\cline { 2 - 3 } & \multicolumn{1}{c}{ Yes } & \multicolumn{1}{c}{ No } \\
\hline $\begin{array}{llr}\text { Changing diagnosis } \\
\text { Yes } \\
\text { No }\end{array}$ & $10^{\mathrm{a}}(62.5)$ & $6(37.5)$ \\
& $12^{\mathrm{a}}(54.5)$ & $10(45.4)$ \\
\hline
\end{tabular}

a. Not statistically significant: $\chi^{2}=0.025, P=0.878$. 
Another common factor among patients with multiple diagnoses was their receiving the diagnosis of personality disorder at some point during their contact with psychiatric services. Also, of the seven patients with a secondary diagnosis of personality disorder, five of them had had multiple changes in diagnosis during the course of their contact. Of the two remaining patients, one had received a primary diagnosis of personality disorder and the other had not previously been in contact with mental health services. Available records did not clearly identify how these diagnoses were made and no evidence of use of any personality disorder questionnaires was found.

\section{Discussion}

The demographic composition of our study sample is comparable with that of patients in other studies of medium secure units, but none of these looked at changes in diagnoses. ${ }^{8,9}$ The patients in our cohort were also similar in terms of their offending and behavioural history and diagnostic distribution. Following assessment at Caswell Clinic, $88.1 \%$ received a diagnosis of a psychotic disorder (78.6\% paranoid schizophrenia) compared with $93 \%$ in the Lelliott et al (2001) survey ${ }^{8}$ and $86 \%$ in the Mckenna (1996) survey. ${ }^{9}$

Our main findings were that over $40 \%$ of patients in this cohort had been subject to changing diagnoses, with an earlier period of recognition of functional psychosis, followed by a period in which substance misuse and/or personality disorder diagnoses prevailed, and finally, under conditions of prolonged, multidisciplinary assessment in the absence of substances (evidenced by negative drug screens), a diagnosis of a functional psychosis was restored, with or without comorbid diagnoses. One of the possible explanations is the natural progression of the illness. Baca-Garcia et al evaluated the long-term stability of the most prevalent psychiatric diagnoses in a variety of clinical settings and found that the percentage of patients retaining their diagnosis in the paranoid schizophrenia group was $69.6 \% .^{10}$ Veen et al looked at diagnostic stability of psychotic disorders and found it to be as high as $91 \% .^{11}$ Both these figures are considerably higher than in our study sample and consequently do not explain the changes that we have noticed.

\section{Comorbid diagnoses}

In our sample, changes in diagnosis were particularly frequent in patients with comorbid alcohol/drug misuse. The comorbidity of substance misuse and mental disorder is well known, and in routine clinical practice patients' mental illness and substance misuse are commonly inexorably intertwined and the cause-effect relationships tend to become very blurred over time. Comorbid mental illness and substance use disorders are often ignored in treatment systems where services specialise in treating either mental disorders or substance use disorders. ${ }^{12}$ In addition, substance use could mask symptoms of mental illness and lead to diagnostic difficulties, which could be a possible explanation for our findings. However, this comorbidity is particularly important as it has been demonstrated repeatedly that schizophrenia with comorbid substance misuse increases the risk of violence considerably compared with schizophrenia without comorbidity. ${ }^{13-16}$ The risk from substance misuse is thought to be additive rather than causative. ${ }^{17}$ The National Confidential Inquiry into suicide and homicide identifies this combination of mental illness and substance misuse as probably the greatest clinical problem facing general adult mental health services and recommends a coordinated approach to training, service planning and research to improve the ability of general services to address this problem. ${ }^{18}$

We also found that changes in diagnosis were more common in patients with a secondary diagnosis of personality disorder compared with those without such a diagnosis. This is understandable given that even in routine presentations it is recognised that the current classification systems for personality disorders are flawed, as the multiple dimensions make it difficult to clearly delineate specific categories. ${ }^{19}$ Given that this cohort of patients presented with multiple presenting symptoms, diagnostic certainty would have been particularly challenging.

Diagnostic uncertainty in this group is particularly relevant as two routes into violence in psychosis are now acknowledged: those who had been unremarkable until the onset of their illness, with an index offence almost invariably reported as driven by psychotic symptoms, and those who had established conduct and/or emotional disorders in childhood, continuous with adult personality disorders who had also developed an illness indistinguishable from schizophrenia. ${ }^{20}$ Clear diagnosis and management in the second group is thus of additional importance and will aid in building strategies for management and prevention of violence. Although the likelihood of violence is in itself not a reason to receive treatment from mental health services, in many cases the diagnosis of personality disorder by itself precludes their involvement. As a result of the diagnosis, some violent patients fall between the stools of mental health services and the criminal justice system, and part of the solution to the problems posed by patients with violent personality disorder is to locate them more firmly within one or the other. ${ }^{21}$

\section{Implications}

Importantly, the findings of this study demonstrate a difference in the management of patients in general adult and forensic settings. Violence/challenging behaviour in an individual with a complex multifactorial presentation is likely to overwhelm the limited resources of general adult services, result in misattribution for the behaviour and hinder detailed in-patient assessments. Forensic settings provide opportunities to conduct prolonged assessments and have facilities to manage these behaviours. Another possible explanation could be that the improved access to detailed and often prolonged in-patient assessments in forensic settings compared with general psychiatry settings may be aiding the diagnostic process in complex multifactorial presentations.

A significant consideration is that 'diagnosis' in psychiatry is a conceptual approach and serves a number of functions, some of which are easily discernible (treatment, management, etc.), whereas others are not. It is recognised 
that there is widespread dislike of patients with personality disorder because of the turmoil they cause in the services, and the prospect of taking them out of a professional group may be welcomed. ${ }^{19}$ Economic pressures with varying degrees of reimbursement to services may also have an impact on the diagnosis assigned. ${ }^{22}$ This evaluation supports the current shift of service inclusion/exclusion criteria being defined by need rather than diagnosis.

The service evaluation was limited by small numbers but has demonstrated that it is likely that there is more diagnostic inconsistency than can be explained by the natural progression of the illness. Unwarranted diagnostic inconsistency means that patients may not receive the proper treatment or access to the right services. It also bears witness to the struggles of professionals and services in trying to provide the right care to patients with complex presentations. Further qualitative research is warranted into whether this is a question of resources or one of service reorganisation. It may also be interesting to compare patients admitted to forensic services with those in general adult services in terms of changing diagnoses.

\section{About the authors}

Dr Amrith Shetty is a consultant psychiatrist at Betsi Cadwaladr University Health Board, Wrexham. Dr Dilum Jayawickrama is a consultant forensic psychiatrist at Partnerships in Care, Llanarth Court, Monmouthshire. Professor Pamela J. Taylor works in the Department of Psychological Medicine and Neurology at Cardiff University.

\section{References}

1 Department of Health. Guidance on the Discharge of Mentally Disordered People and their Continuing Care in the Community. HMSO, 1994.

2 Taylor PJ, Gunn J. Diagnosis, medical models and formulations. In Handbook on Forensic Mental Health (eds K Soothhill, M Dolan, P Rogers): 268-97. William Publishers, 2008.

3 Spokes J. Report of the Committee of Inquiry into the Care and Aftercare of Miss Sharon Campbell. HMSO, 1988

4 Ritchie JH, Dick D, Lingham R. The Report of the Inquiry into the Care and Treatment oj Christopher Clunis. HMSO, 1994.

5 Healthcare Inspectorate Wales. Report of a Review in Respect of Ms A and the Provision of Mental Health Services, Following a Homicide Committed in October 2005. Healthcare Inspectorate Wales, 2008 (http:// www.hiw.org.uk/Documents/477/cardiff\%20homicide\%20for\%20 print\%20final2.pdf).
6 Melzer D, Tom BDT, Brugha T, Fryers T, Gatward R, Grounds A, et al. Access to medium secure psychiatric care in England and Wales: a national survey of admission assessments. J Forensic Psychiatry Psychol 2009; 15: 7-31.

7 NHS National Patient Safety Agency (NSPA). Defining Research. National Research Ethics Service, NPSA, 2010 (http://www.nres. nhs.uk/news-and-publications/publications/general-publications).

8 Lelliott P, Audini B, Duffett R. Survey of patients form an inner-London health authority in medium secure psychiatric care. Br J Psychiatry 2001; 178: 62-6.

9 McKenna J. In-patient characteristics in a regional secure unit. Psychiatr Bull 1996; 20: 264-8.

10 Baca-Garcia E, Perez-Rodriguez MM, Basurte-Villamor I, Fernandez Del Moral AL, Jimenez-Arriero MA, Gonzalez De Rivera JL, et al. Diagnostic stability of psychiatric disorders in clinical practice. Br J Psychiatry 2007; 190: $210-6$.

11 Veen ND, Selten J-P, Schols D, laan W, Hoek HW, van der Tweel I, et al. Diagnostic stability in a Dutch psychosis incidence cohort. $\mathrm{Br} J$ Psychiatry 2004; 185: 460-4.

12 Hall W, Farrell M. Comorbidity of mental disorders with substance misuse. Br J Psychiatry 1997; 171: 4-5.

13 Swanson JW, Borum R, Swartz MS. Psychotic symptoms and disorders and the risk of violent behaviour in the community. Crim Behav Ment Health 1996; 6: 309-29.

14 Cuffel BJ, Shumway M, Choujian TL, MacDonald T. A longitudinal study of substance use and community violence in schizophrenia. J Nerv Ment Dis 1994; 182: 704-8.

15 Tiihonen J, Isohanni M, Rasanen P, Koiranen M, Moring J. Specific major mental disorders and criminality: a 26-year prospective study of the 1966 northern Finland birth cohort. Am J Psychiatry 1997; 154: 840-5.

16 Wallace C, Mullen P, Burgess P, Palmer S, Ruschena D, Browne C. Serious criminal offending and mental disorder. Case linkage study. $\mathrm{Br} \mathrm{J}$ Psychiatry 1998; 172: 477-84.

17 Walsh E, Buchanan A, Fahy T. Violence and schizophrenia: examining the evidence. Br J Psychiatry 2002; 180: 490-5.

18 Department of Health. Safety First: Five-Year Report of the National Confidential Inquiry into Suicide and Homicide by People with Mental Illness. TSO (The Stationery Office), 2001.

19 Manning N. DSM-IV and dangerous and severe personality disorder an essay. Soc Sci Med 2006; 63: 1960-71.

20 Taylor PJ. Second Expert Paper: Mental IIIness and Serious Harm to Others. NHS National Programme on Forensic Mental Health Research and Development, 2007 (http://www.liv.ac.uk/fmhweb/EP\%20Mental\%20 Illness\%20and\%20Serious\%20Harm \%20to\%20Others.pdf).

21 Appleby L. Safer services: conclusions from report of the National Confidential Inquiry. Adv Psychiatr Treat 2000; 6: 5-15.

22 Stone $\mathrm{MH}$. Relationship of borderline personality disorder and bipolar disorder. Am J Psychiatry 2006; 163: 1126-8. 